\title{
Trackhold: a Novel Passive Arm-Support Device
}

\author{
Basilio Lenzo \\ Perceptual Robotics Laboratory \\ TeCIP, Scuola Superiore Sant'Anna \\ Via Alamanni 13/B 56017 Ghezzano \\ Pisa, Italy \\ Email: b.lenzo@sssup.it
}

\author{
Simone Marcheschi \\ Perceptual Robotics Laboratory \\ TeCIP, Scuola Superiore Sant'Anna \\ Via Alamanni 13/B 56017 Ghezzano \\ Pisa, Italy \\ Email: s.marcheschi@sssup.it
}

\author{
Antonio Frisoli \\ Perceptual Robotics Laboratory \\ TeCIP, Scuola Superiore Sant'Anna \\ Via Alamanni 13/B 56017 Ghezzano \\ Pisa, Italy \\ Email: a.frisoli@sssup.it
}

\author{
Marco Fontana \\ Perceptual Robotics Laboratory \\ TeCIP, Scuola Superiore Sant'Anna \\ Via Alamanni 13/B 56017 Ghezzano \\ Pisa, Italy \\ Email:m.fontana@sssup.it
}

Fabio Salsedo

Perceptual Robotics Laboratory

TeCIP, Scuola Superiore Sant'Anna

Via Alamanni 13/B 56017 Ghezzano

Pisa, Italy

Email: f.salsedo@sssup.it

\author{
Massimo Bergamasco \\ Perceptual Robotics Laboratory \\ TeCIP, Scuola Superiore Sant'Anna \\ Via Alamanni 13/B 56017 Ghezzano \\ Pisa, Italy \\ Email: m.bergamasco@sssup.it
}

This article introduces the design and the experimental validation of the Trackhold, a novel mechanical motion-tracker for upper limb physical rehabilitation. The Trackhold is based on a passively balanced mechanism that can approximately relieve the weight of the patient's arm regardless of the position. The system features a novel kinematic architecture with large workspace and custom developed joint sensors providing accurate real-time measure of the upper limb posture. The design approach of the device, which went through kineto-static and dynamic analyses, is presented and details on the employed mechatronic solutions are illustrated. Lastly, a functional validation of a prototype of the Trackhold is provided.

\section{Introduction}

People affected by neuromuscular disorders present limited ability to perform basic Activities of Daily Living (ADL). In several cases, depending on the type and severity of the pathology, it is possible to fully or partially recover the loss functionalities through rehabilitation therapy.

Conventional rehabilitation therapy consists in practicing exercises with the support of a specialized medical doctor (physical therapists) that supervises and physically facilitates the prescribed limb movements. The success of such rehabilitation procedures is strongly affected by the amount of time/repetitions of therapeutic procedures and by the subjective abilities of the physical therapist [1-4].

In the last years, with the aim of providing more uniform/constant and frequent therapeutic physical rehabilitation, several researchers have investigated the possibility for such therapies to be automated $[5,6]$. Specifically, a branch of research on rehabilitation engineering is focusing on the development of robotic/mechatronic devices that could help impaired people to reuse their limbs practicing arm movements without continuous supervision from a rehabilitation therapist. Such systems are designed to exert desired forces on the user's limbs to facilitate the execution of movements that are prescribed by rehabilitation therapy and most of them are focussed on arm rehabilitation.

Robotic/mechatronic devices for arm rehabilitation can be of active or passive nature. Active devices include actuators that deliver controlled power/forces on the user's body, while passive systems make use of special designed mechanisms to provide passively controlled forces.

In active systems, the arm programmed forces are provided by exoskeletons or endpoint manipulators [7]. Exoskeletons are wearable robots having the same (or similar) kinematics of the human arm, like ABLE [8], L-EXOS [9] and ARMEO [10]. Endpoint manipulators have a single connection to the hand, wrist, or forearm with which patients make planar or 3D movements, like ARMON [11]. Sim- 
pler devices can be passive systems without actuators, showing more limited functionalities but with advantages in cost and simplicity in use/maintenance with respect to active systems. A typical aid provided by passive systems is the arm weight compensation, i.e. the balancing or the minimization of the effort that is required by the patient to hold her/his arm against gravity. This type of compensation plays a key role, as it helps the execution of several types of arm movement and it can provide significant improvements in the arm function of patients $[6,10,12-14]$. The use of such devices is classified as AAROM exercise (Active assistive range of motion), since the user is actively moving his limb, with an external aid [15].

Several passive system have been developed in the last years. A cable system is introduced in the FREEBAL system [16], in which the arm weight is compensated by a suspension cable that exerts an approximately a constant vertical force. A different approach based on a serial mechanism with a spring system is proposed by [11]: the vertical force that compensates the arm weight is provided by clever arrangements of springs that allow to exert a constant vertical force on the forearm of the user. Hybrid solutions, i.e. passive systems that are also able to slowly vary the intensity and direction of the weight compensation force, have been also investigated [17].

In this paper, we present the Trackhold, a novel passive arm-support device that shows some different features with respect to existing devices. Specifically, the Trackhold: i) presents an arm weight compensation system based on counterweights instead of more commonly employed springs; ii) integrates a 6 Degrees of Freedom (DoFs) sensing system that tracks the position and the orientation of the user's arm in realtime. The first feature has been driven by the practical requirement (discussed with different physical therapists) of being able to rapidly change the intensity of the compensation force when switching from a patient to another. However counterweights, differently from springs, add a significant inertia contribution to the device: the effect of this is analysed later in the paper. The second feature has been introduced in order to be able to record the arm movements during the therapy and/or to make it possible to connect the system to interactive computer based environment (such as Virtual Reality systems), that can help the deployment of rehabilitation procedures.

The design of the Trackhold is based on a novel kinematic configuration that is capable to achieve an approximately static arm weight balancing in a large 3D workspace. An analytical model of the device is then employed to assess the performance of the system under dynamic conditions. Analytical simulations and verification based on experimental measures with healthy subjects are also provided. The realized prototype of the Trackhold employs a special designed low-cost sensing system based on a hall-effect magnetic sensor that makes it possible to achieve a compact mechanical design with fully enclosed electrical cabling.

The paper is structured as follows. Section 2 concerns the kineto-static design of the Trackhold. Section 3 describes the theoretical verification of the dynamic behaviour of the

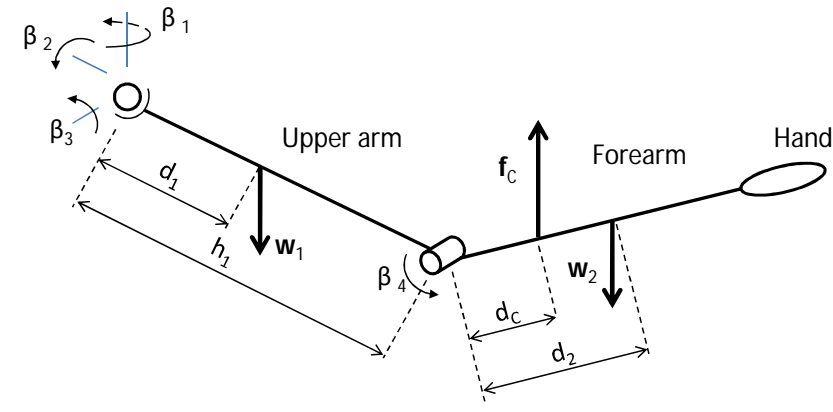

Fig. 1. Human arm's kinematics and CCM principle

device. Section 4 reports details on the integration of the device. Section 5 reports the setup and results of the experimental test. Conclusion is in Section 6.

\section{Kineto-static design}

The Trackhold is conceived to measure position and orientation in space of the patient's arm (Track) and, at the same time, provide static balancing of the patient's arm weight regardless of the configuration (Hold). The Trackhold is based on a passive mechanism that employs compensation masses, dimensioned to exert appropriate forces on the user forearm and to substantially reduce the tonic muscle contraction required to hold her/his arm against gravity.

As a guideline for the design, the following qualitative requirements have been considered:

- the device is intended for a user sitting on a chair;

- the reachable workspace of the device must be comparable to that of human arm;

- the device should not interfere with the user limb in all its workspace;

- the user's visual field should be as much free as possible.

\subsection{Arm-weight balancing principle (CCM)}

The working principle is based on the concept of Combined Center of Mass (CCM), which allows to balance the arm weight applying an external force in just one point. In order to provide an explaination of such a working principle we consider the human arm scheme shown in Fig. 1. Specifically, the elbow joint is represented as a purely rotational joint and the glenohumeral joint is modelled with a spherical joint. This generally represents a good approximation of the shoulder kinematics, that is particularly well acceptable for purpose of deriving a balancing model of weight since, as it is shown in the following, the derivation of the balancing equations is not affected by other movements of the shoulder joint, like scapula depression or elevation, retraction and protraction. The upper arm and the forearm are represented as rigid bodies having their center of mass respectively at distance $d_{1}$ and $d_{2}$ from the shoulder and elbow joints.

The vector of the torques $\tau$ required at the joints to en- 
sure the static equilibrium is given by the following [18]

$$
\tau=\mathbf{J}_{1}^{T} \mathbf{w}_{1}+\mathbf{J}_{2}^{T} \mathbf{w}_{2}+\mathbf{J}_{C}^{T} \mathbf{f}_{C}
$$

where $\mathbf{w}_{1}=\left[\begin{array}{lll}0 & 0 & m_{1} g\end{array}\right]^{T}$ is the weight force of the upper arm, $\mathbf{w}_{2}=\left[\begin{array}{lll}0 & 0 & m_{2} g\end{array}\right]^{T}$ is the weight force of the forearm and $\mathbf{f}_{C}=\left[\begin{array}{lll}0 & 0 & f_{C}\end{array}\right]^{T}$ is the balancing force that has a vertical upward constant direction and is applied at a point (CCM point) that lays on a plane that is normal to the elbow joint and pass through the center of mass of the forearm at a distance $d_{C}$ from the elbow; $\mathbf{J}_{1}, \mathbf{J}_{2}$ and $\mathbf{J}_{C}$ are the Jacobian matrices relative to each application point of the forces, obtained according to [18] and without considering the first joint of the kinematic chain, ${ }^{1}$ which is parallel to all the forces acting on the system and hence has no effect on the static equilibrium analysis $\left(\tau_{1}=0\right)$. In detail, it results:

$$
\tau=\left[\begin{array}{c}
\tau_{2} \\
\tau_{3} \\
\tau_{4}
\end{array}\right]=\left[\begin{array}{c}
\left(w_{2} d_{2}-f_{C} d_{C}\right) s_{\beta_{2}} s_{\beta_{34}}+\left(w_{2} h_{1}+w_{1} d_{1}-f_{C} h_{1}\right) s_{\beta_{2}} s_{\beta_{3}} \\
-\left(w_{2} d_{2}-f_{C} d_{C}\right) c_{\beta_{2}} c_{\beta 34}-\left(w_{2} h_{1}+w_{1} d_{1}-f_{C} h_{1}\right) c_{\beta_{2}} c_{\beta_{3}} \\
c_{\beta_{2}} c_{\beta 34}\left(f_{C} d_{C}-w_{2} d_{2}\right)
\end{array}\right]
$$

$$
\mathbf{J}_{1}=\left[\begin{array}{ccc}
-d_{1} c_{2} s_{\beta_{3}} & -d_{1} c_{\beta_{3}} s_{\beta_{2}} & 0 \\
-d_{1} s_{\beta_{2}} s_{\beta_{3}} & d_{1} c_{\beta_{2}} c_{\beta_{3}} & 0 \\
0 & -d_{1} s_{\beta_{3}} & 0 \\
0 & -c_{\beta_{2}} & 0 \\
0 & -s_{\beta_{2}} & 0 \\
1 & 0 & 0
\end{array}\right]
$$

$\mathbf{J}_{2}=\left[\begin{array}{ccc}-c_{\beta_{2}}\left(d_{2} s_{\beta_{34}}+h_{1} s_{\beta_{3}}\right) & -s_{\beta_{2}}\left(d_{2} c_{\beta_{34}}+h_{1} c_{\beta_{3}}\right) & -d_{2} c_{\beta_{34}} s_{\beta_{2}} \\ -s_{\beta_{2}}\left(d_{2} s_{\beta_{34}}+h_{1} s_{\beta_{3}}\right) & c_{\beta_{2}}\left(d_{2} c_{\beta_{34}}+h_{1} c_{\beta_{3}}\right) & d_{2} c_{\beta_{34}} c_{\beta_{2}} \\ 0 & -d_{2} s_{\beta_{34}}-h_{1} s_{\beta_{3}} & -d_{2} s_{\beta_{34}} \\ 0 & -c_{\beta_{2}} & -c_{\beta_{2}} \\ 0 & -s_{\beta_{2}} & -s_{\beta_{2}} \\ 1 & 0 & 0\end{array}\right]$

$$
\mathbf{J}_{C}=\left[\begin{array}{ccc}
-c_{\beta_{2}}\left(d_{C} s_{\beta_{34}}+h_{1} s_{\beta_{3}}\right) & -s_{\beta_{2}}\left(d_{C} c_{\beta_{34}}+h_{1} c_{\beta_{3}}\right) & -d_{C} c_{\beta_{34}} s_{\beta_{2}} \\
-s_{\beta_{2}}\left(d_{C} s_{\beta_{34}}+h_{1} s_{\beta_{3}}\right) & c_{\beta_{2}}\left(d_{C} c_{\beta_{34}}+h_{1} c_{\beta_{3}}\right) & d_{C} c_{\beta_{34}} c_{\beta_{2}} \\
0 & -d_{C} s_{\beta_{34}}-h_{1} s_{\beta_{3}} & -d_{C} s_{\beta_{34}} \\
0 & -c_{\beta_{2}} & -c_{\beta_{2}} \\
0 & -s_{\beta_{2}} & -s_{\beta_{2}} \\
1 & 0 & 0
\end{array}\right]
$$

where we use the conventions $x=|\mathbf{x}|$ for the generic vector $\mathbf{x}, s_{\beta_{i}}=\sin \beta_{i}, c_{\beta_{i}}=\cos \beta_{i}, s_{\beta_{i j}}=\sin \left(\beta_{i}+\beta_{j}\right)$ and $c_{\beta_{i j}}=$ $\cos \left(\beta_{i}+\beta_{j}\right)$.

The balancing conditions $\tau_{i}=0$ for $i=1 \ldots 4$ regardless of the configuration $\beta_{i}$ gives

$$
\mathbf{f}_{C}=-\left(\mathbf{w}_{2}+\mathbf{w}_{1} \frac{d_{1}}{h_{1}}\right)
$$

\footnotetext{
${ }^{1}$ The spherical joint can be thought of as a series of three rotational joints with mutually orthogonal and incident axes.
}

and

$$
d_{C}=\frac{d_{2}}{1+\frac{m_{1} d_{1}}{m_{2} h_{1}}}
$$

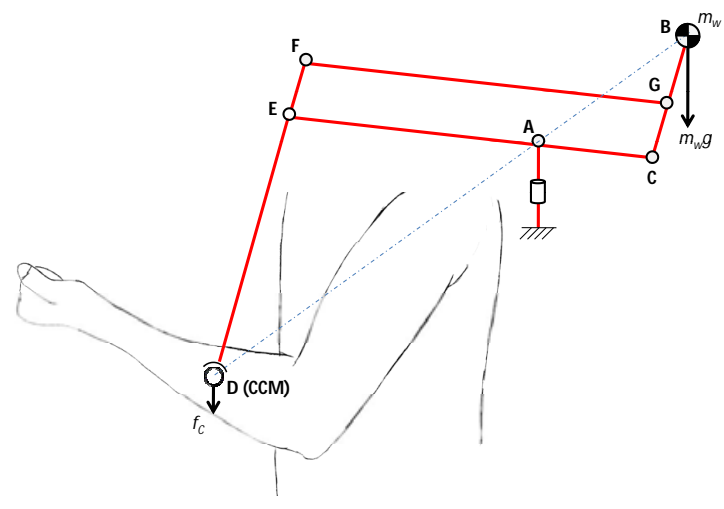

Fig. 2. Pantograph and counterweight implementing the CCM

So, the exertion of a vertical upward force at the CCM point makes it possible to achieve a perfect static balance of a human arm regardless of the configuration. The actual amount of the the patient's arm mass supported by the device is around $75 \%$ (see Eq. 6 and consider that $m_{1} \approx m_{2}$ and $d_{1} \approx h_{1} / 2$ ), in a single supporting point. Some experiments already demonstrated that the CCM approach could be effectively applied to static arm balancing [11].

Practical values of the vertical force $\mathbf{f}_{C}$ and of the parameter $d_{C}$ have been evaluated considering average mass and center of gravity positions of upper arm and forearm detailed in [19]. Moreover, a brief experimental verification was conducted within a group of 6 healthy subjects ( 4 males, 2 females) using a simple setup made of a forearm elastic band attached to a cable that exerted the pulling force through a pulley and a balancing weight. Experimental tests consisted of setting a fixed distance ${ }^{2} d_{c}$ and increasing the balancing weight from 0.4 to $4 \mathrm{~kg}$ ( $0.25 \mathrm{~kg}$ steps). The resulted range of ideal weights was between 1.15 and $2.9 \mathrm{~kg}$, corresponding respectively to a $54 \mathrm{~kg}$ and a $98 \mathrm{~kg}$ person.

\subsection{Implementation of the CCM}

The practical implementation of the CCM principle requires to exert a vertical force on a specific point of the user's forearm, such a force remaining constant (both in magnitude and direction) regardless of the movement of the arm.

As shown in Fig. 2, this has been implemented through a pantograph mechanism $E F G C$ having two elongated links defining points $D$, which is the CCM point, and $B$, where an

\footnotetext{
${ }^{2} d_{c}=120 \mathrm{~mm}$, considering Eq. 7 with $m_{1} \approx m_{2}, d_{2} \approx d_{1}, d_{1} \approx h_{1} / 2$ and $h_{1} \approx 360 \mathrm{~mm}$ [19].
} 


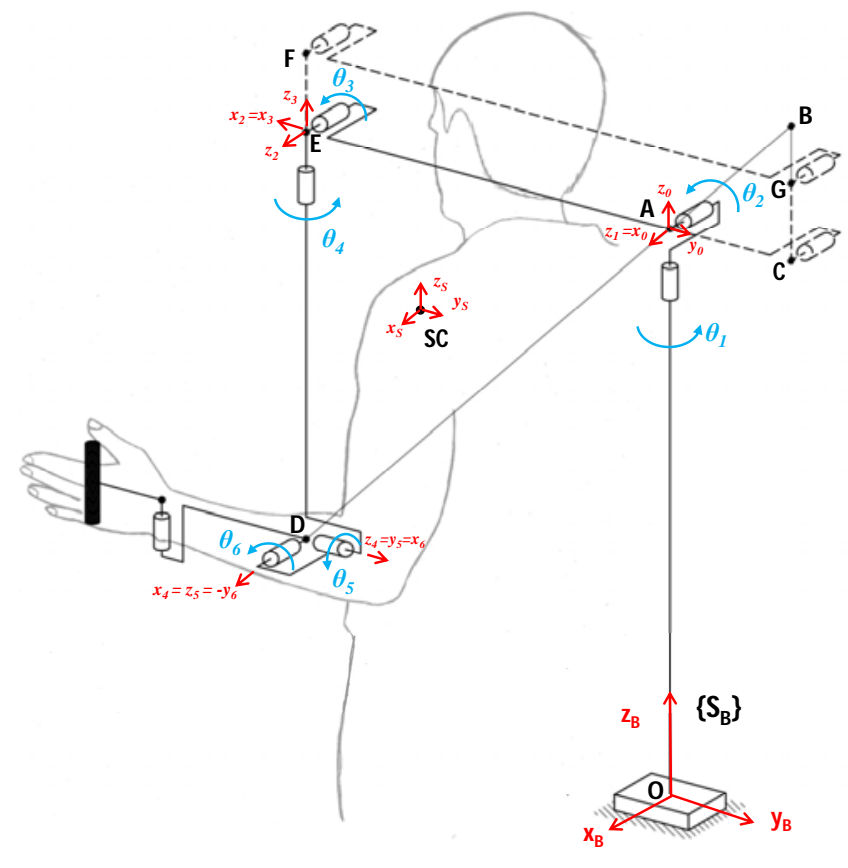

Fig. 3. Kinematics of the Trackhold

adjustable counterweight $m_{w}$ is located. ${ }^{3}$ With respect to the joint in point $A$ (which connects the pantograph to the frame), the CCM point and the counterweight $m_{w}$ lay on opposite sides. Being the two segments $C G$ and $E F$ parallel (due to the pantograph kinematics), we chose $A C / B C=A E / D E$ obtaining that the triangles $A B C$ and $A D E$ are similar. As a result, the two segments $A B$ and $A D$ are aligned independently of the position of the mechanism, then the supporting force $\mathbf{f}_{C}$ at the CCM point can be produced with a counterweight mass $m_{w}$ at point $B$ defined by $m_{w} g=f_{c} A D / A B=f_{c} A E / A C$. To limit the encumbrance of the passive balancing system, we chose $A B / A D<1$. On the other hand, $A B / A D$ should not be too small so as to avoid large values of $m_{w}$ which produces a undesired added inertia to the system (see Section 3).

\subsection{Kinematics}

As regards the complete kinematic structure, we chose a six Degrees of Freedom (DoFs) serial chain featuring six rotational joints connected by rigid links. Figure 3 shows the kinematics of the Trackhold where the six joint coordinates are denoted with $\theta_{i}(i=1$ to 6$)$ and the pantograph mechanism $(E F G C)$ has been included.

The direct and inverse kinematics of the whole device were modelled in Matlab, in which the six joint angles $\theta_{i}$ were used as a set of lagrangian coordinates. The coordinate frames were selected according to the Denavit-Hartenberg (DH) convention [20]: DH parameters are reported in Tab. 1, where $A E=D E=l_{2}=l_{4}$. The position vector $\mathbf{p}$ of the CCM point, namely point $D$, with respect to the ground (base frame $S_{B}$ in Fig. 3), is calculated using the direct kinematics of the

\footnotetext{
${ }^{3}$ Two additional counterweights $m_{w_{B}}$ and $m_{w_{C}}$ applied respectively in points $B$ and $C$ in order to balance the weight of the pantograph itself.
}

device:

$$
\mathbf{p}=l_{2}\left[\begin{array}{c}
-s_{\theta_{1}} c_{\theta_{2}} s_{\theta_{3}}+s_{\theta_{1}} c_{\theta_{2}}-s_{\theta_{1}} s_{\theta_{2}} c_{\theta_{3}} \\
c_{\theta_{1}} c_{\theta_{2}} s_{\theta_{3}}-c_{\theta_{1}} c_{\theta_{2}}+c_{\theta_{1}} s_{\theta_{2}} c_{\theta_{3}} \\
s_{\theta_{2}} s_{\theta_{3}}-c_{\theta_{2}} c_{\theta_{3}}-s_{\theta_{2}}+a / l_{2}
\end{array}\right]
$$

Table 1. Denavit-Hartenberg Table for the Trackhold

\begin{tabular}{c||c|c|c|c} 
Link & $a$ & $\alpha$ & $d$ & $\theta$ \\
\hline 1 & 0 & $\pi / 2$ & 0 & $\theta_{1}$ \\
2 & $l_{2}$ & 0 & 0 & $\theta_{2}+\pi$ \\
3 & 0 & $\pi / 2$ & 0 & $\theta_{3}$ \\
4 & 0 & $-\pi / 2$ & $-l_{4}$ & $\theta_{4}+\pi / 2$ \\
5 & 0 & $-\pi / 2$ & 0 & $\theta_{5}+\pi / 2$ \\
6 & 0 & $-\pi / 2$ & 0 & $\theta_{6}+\pi / 2$
\end{tabular}

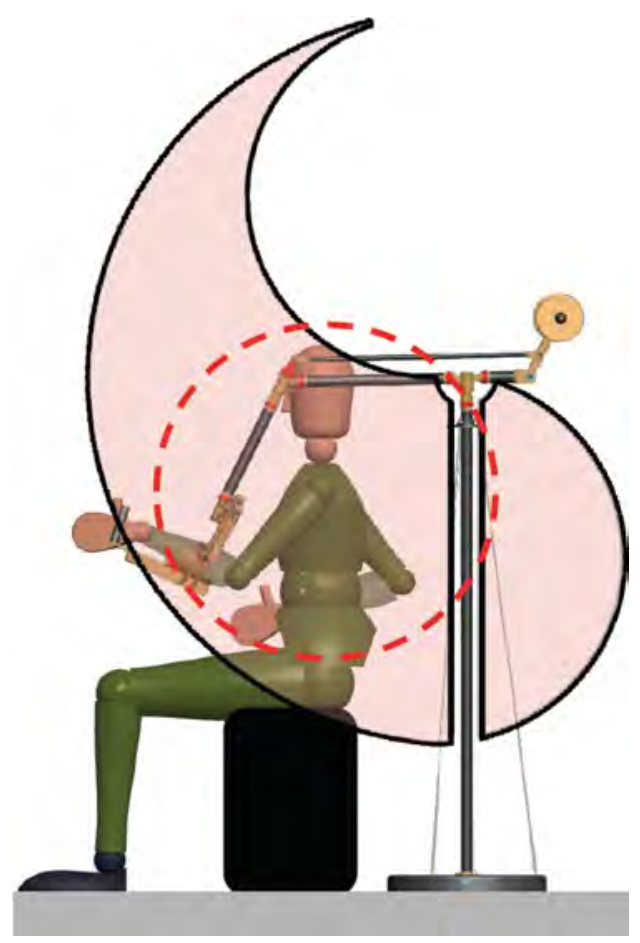

Fig. 4. Trackhold workspace, referred to the CCM point reachable positions, compared with an approximate representation of the human arm workspace

where $a=O A, s_{\theta_{i}}=\sin \theta_{i}, c_{\theta_{i}}=\cos \theta_{i}, s_{\theta_{i j}}=\sin \left(\theta_{i}+\right.$ $\left.\theta_{j}\right)$ and $c_{\theta_{i j}}=\cos \left(\theta_{i}+\theta_{j}\right)$. The orientation of the user's arm is also obtained via direct kinematics, and it is defined as the orientation of the final reference frame, $\left\{x_{6}, y_{6}, z_{6}\right\}$, with respect to the base frame $S_{B}$. 
The position of the joints and the dimensions of the links have been chosen based on general considerations and trade offs between the need of a large workspace (in order to cover most of the workspace of the human arm [21]), the need of avoidance of collisions with the human during the movement of the device, and the need of a device compact and portable.

The first joint of the Trackhold is located behind the user: referring to Fig. 3, the coordinates of point $A$ with respect to the shoulder center $S C$ are, in the $\left\{x_{s}, y_{s}, z_{s}\right\}$ frame, $[0,280,425] \mathrm{mm}$. The fifth joint $\left(\theta_{5}\right)$ has been realized by means of a remote center of motion mechanism (RCM). A $\mathrm{RCM}$ is a 1 DoF mechanism that implements a purely rotational joint while its mechanical structure is delocalized from the rotation axis. Several possible layouts and schemes are reported in [22] and [23]. In the application of this paper, the RCM makes the space around the axis to be free to host the user forearm. A section (along a vertical plane) of the workspace of the manipulator compared with an approximate representation of the human arm workspace is provided in Fig. 4.

\section{Theoretical dynamic verification}

The passive gravity balancing approach does not take into account dynamic effects. Then, it is important to verify that the effects due to the inertia of the device do not affect its performance in terms of exerted force and user's feeling. In order to have a quantitative estimation of disturbing forces that are introduced by the inertia of the device, we employ a procedure for dynamics verification that consists in i) building up a dynamic model of the Trackhold (Section 3.1); ii) experimentally acquiring trajectories representing sample movements of the end effector during the use of the device (Section 3.2); iii) imposing such trajectories in the dynamic model at the level of the end effector (Section 3.3) and analyse the output. As a result, the dynamic model provides the accelerations and the interaction force $\mathbf{F}$ exerted by the device on the user during the imposed movements. The interaction force $\mathbf{F}$ can be split into vector components $F_{i}$, being $i=$ $1,2,3$ respectively the components along $x_{0}, y_{0}, z_{0}$ (which are the axes of the first frame of the kinematic chain, defined in 2.3, see Fig. 3). Further, each component can be written as $F_{i}=F_{i}^{s}+F_{i}^{d}$, expliciting the static contribution $F_{i}^{s}$ (due to mass of the counterweights) and the dynamic contribution $F_{i}^{d}$.

As a measure of the good performance of the Trackhold, we expected:

- regarding the exerted force, a $F_{i}$ close to the theoretical value, with oscillations limited to $10-15 \%$;

- regarding the user's feeling, defining $m_{i j}=F_{i}^{d} / a_{j}$, a constant value for each $m_{i j}$ when $i=j$, while $m_{i j}=0$ for $i \neq j$, meaning that the dynamic effect of using the Trackhold is comparable to an added mass at the level of the end effector, not entailing unnatural feelings for the user.

\subsection{Model of the Trackhold}

The dynamics of the device was modelled in Matlab, according to the classical notation [18]:

$$
\mathbf{B}(\mathbf{q}) \ddot{\mathbf{q}}+\mathbf{C}(\mathbf{q}, \dot{\mathbf{q}}) \dot{\mathbf{q}}+\mathbf{G}(\mathbf{q})=\tau(\mathbf{F}, q)
$$

being $\mathbf{q}$ the coordinates of the six joints (lagrangian coordinates) and $\tau$ the torques at the level of the joints resulting from the application of the external wrench $\mathbf{F}$ at the end effector (interface point). It is $\tau=-\mathbf{J}_{H}^{T} \mathbf{F}, \mathbf{J}_{H}$ being the Jacobian related to the interface point (point D in Fig. 3).

Further details on the dynamic model of the Trackhold are reported in Appendix 6.

\subsection{Optical acquisitions setup}

As stated at the beginning of this Section, the dynamic verification of the Trackhold was carried out imposing predetermined trajectories to the end effector and analyzing the related inertial effect on the performance of the device. Such trajectories were experimentally obtained by optical acquisitions of the movements of six healthy subjects asked to execute a simple task with their arm. The task was required to be in a restricted workspace, to be sure that the acquired trajectories were inside the workspace of the Trackhold. The test setup is shown in Fig. 5, where the subject can move his forearm in a horizontal plane above a planar clock-like target. Each subject was instructed to perform an outward movement from the center to a random point among the extremities on the external circumference, and another movement back to the center. The two movements were repeated for one minute, at a frequency given by a metronome set to 50 beats per minute ( 1 beat per movement). The arm movements were recorded by a camera-based optical 3D tracking system, NaturalPoint Optitrack [24]. The output data were the position and orientation of a coordinate frame having origin in the point corresponding to the human-device interaction point (CCM), where three proper reflective markers were attached (see Fig. 5). Based on this, the joint positions over time were computed through the inverse kinematics model of the Trackhold. Such data were used as the input for the developed dynamic model of the device, allowing to compute the wrench $\mathbf{F}$ exerted at the interaction point.

\subsection{Simulation results}

The numerical values adopted in this analysis are $a=$ $O A=1477.25 \mathrm{~mm}, A B / A D=1 / 3$ and $A E=D E=l_{2}=l_{4}=$ $525 \mathrm{~mm}$, which correspond to the practical values adopted in the final design. Two supporting force values were used, respectively $11.30 \mathrm{~N}(1.15 \mathrm{~kg})$ and $28.5 \mathrm{~N}(2.9 \mathrm{~kg}){ }^{4}$ According to the frame $\left\{x_{0}, y_{0}, z_{0}\right\}$ (Fig. 3 ), $z$ is the axis aligned with the gravity direction, i.e. the axis where the force support is expected.

\footnotetext{
${ }^{4}$ These are the minimum and maximum values resulting from the experiment reported in 2.1 .
} 
Figure 6 and Fig. 7 show the results of the simulation for one representative subject and $11.30 \mathrm{~N}$ supporting force: i) the recorded position of the reflective target is, as expected, a clock-like shape; ii) the average force for the five subjects on the $z$ axis resulted $11.82 \pm 1.12 \mathrm{~N}$, close to the theoretical value $(11.30 \mathrm{~N})$; iii) forces along axes $x$ and $y$ were respectively $0.20 \pm 2.07 \mathrm{~N}$ and $0.07 \pm 2.80 \mathrm{~N}$; iv) torques along axes $x, y$ and $z$ resulted, respectively, below $0.4 \mathrm{Nm}, 0.2 \mathrm{Nm}$ and $0.1 \mathrm{Nm}$; v) the average $m_{i j}$ for $\mathrm{i}=\mathrm{j}$ resulted $5.15 \pm 0.88 \mathrm{~kg}$, while it resulted $6.40 \mathrm{~kg}$ as an average over all the subjects; vi) the spurious contributions $\left(m_{i j}, i \neq j\right)$ resulted always less than $0.5 \mathrm{~kg}$. For the case of $28.5 \mathrm{~N}$ supporting force, the average force for the five subjects on the $z$ axis resulted $28.94 \pm 1.13 \mathrm{~N}$, while the other results changed less than $9 \%$. This means that the inertial contribution of the counterweights generating the supporting force ${ }^{5}$ is practically negligible. Nonetheless, the weight of the device without such counterweights is around $21 \mathrm{~kg}$.

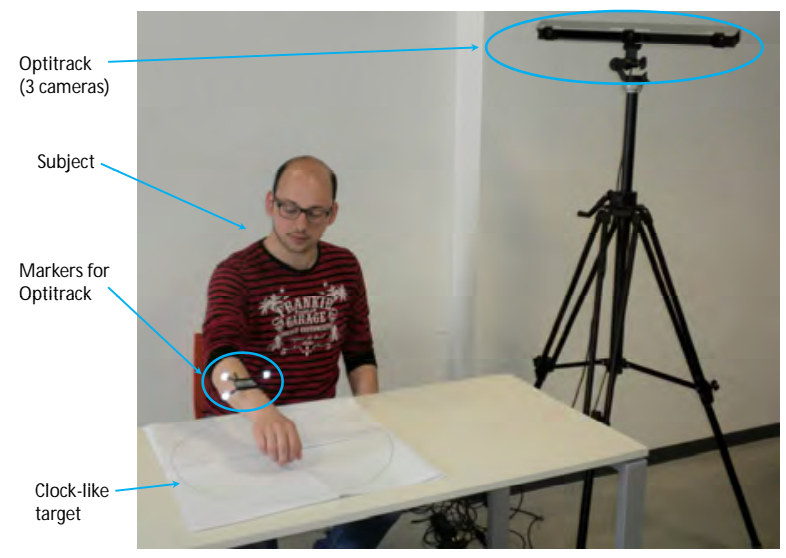

Fig. 5. Test for the dynamic analysis of the Trackhold

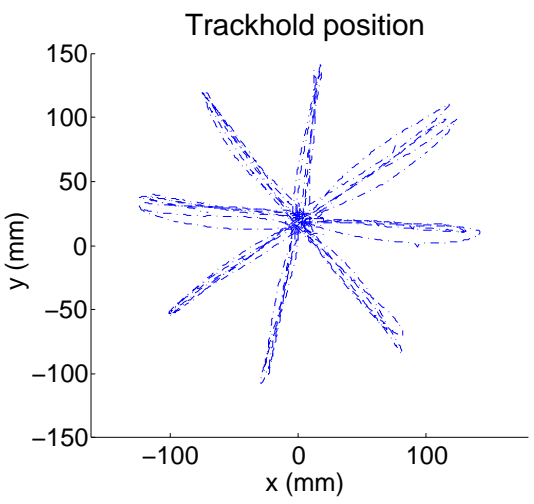

Fig. 6. Recorded target position (subject 4)

\footnotetext{
${ }^{5}$ As described in 2.2, there are also some counterweights used to balance the structure of the Trackhold.
}

The above simulation refer to movements along the horizontal plane, as stated in Section 3.2. As a further check, other simulations were performed imposing the acquired trajectories on the other two planes. This means including movements along the vertical axis, where higher inertia contribution are expected. Indeed, the only significant change in the results is an increase by $\approx 40 \%$ of the standard deviation of the exerted vertical force (e.g. for the case of $11.30 \mathrm{~N}$ supporting force the simulation result is $11.82 \pm 1.59 \mathrm{~N}$ against $11.82 \pm 1.12 \mathrm{~N}$ in the horizontal plane).

To sum up, the results of this analysis showed that the Trackhold exerts the desired force with $\mathrm{a} \approx 10 \%$ uncertainty, while the dynamic effect for the user is comparable to an added mass at the level of the interaction point, endorsing a natural feeling for the user throughout all the spatial directions. This was further confirmed analyzing separately the inertial contributions and the centrifugal-Coriolis contributions during the simulation, the latter resulting negligible with respect to the former.

\section{Detailed integration of the device}

In light of the results of the previous analysis, the Trackhold was completely designed, fabricated and assembled. Specific design solutions have been thought and implemented for both mechanics and electronics.
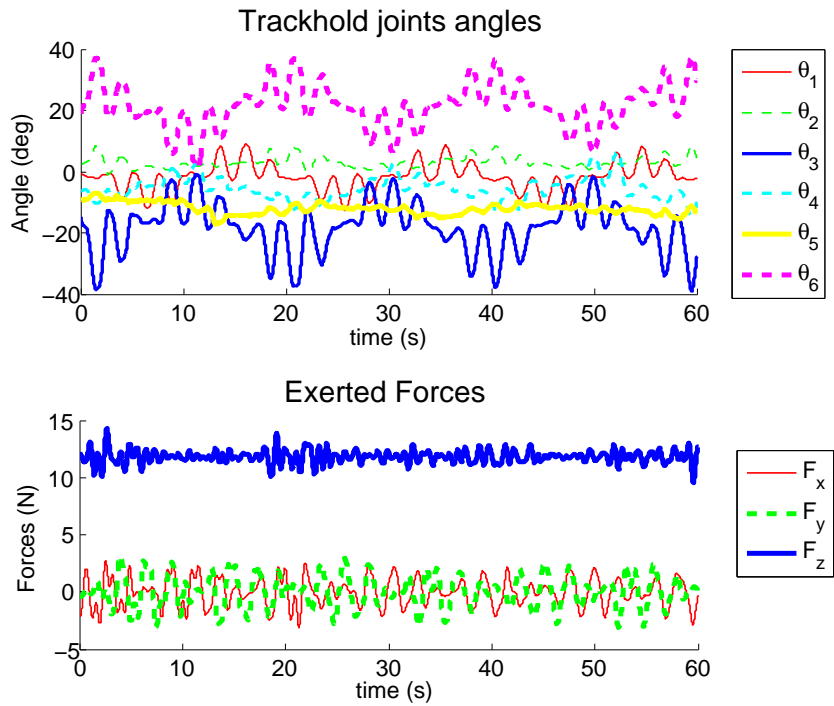

Fig. 7. Simulated joint coordinates and wrench exerted to the user (subject 4)

\subsection{Kinematic implementation}

The first four DoFs are obtained combining two sensorized flexion-torsion joints, that is a compact implementation of two serial rotational joints having orthogonal incident axes (see Section 4.2). The fifth DoF allows the pronosupination of the forearm, namely the rotation of the forearm 
with respect to its longitudinal axis. This DoF is realized by means of a remote center of rotation mechanism (RCM), as described in Section 2.3. The sixth DoF, allowing the elbow flexion, is achieved by means of a standard rotational joint.

The first joint is linked to ground by a hollow cylindrical tube and a wheeled base, which supports all the structure. The two flexion-torsion joints are linked by another hollow cylindrical tube. The device can support either the left or right arm of the user, due to a simple swapping system for the forearm links. The workspace of the device could be easily scaled changing the length of the tubes.

The CAD model of the Trackhold is in Fig. 8, which shows the device without external covers and a user using the device with external covers.

\subsection{Modular flexion-torsion joint}

The modular flexion-torsion joint is a two DoF modular unit made out of two orthogonal rotational joints. It is an integrated system that provides several features: it is a structural element realizing the desired kinematics, it provides sensing and it allows cable layout routing.

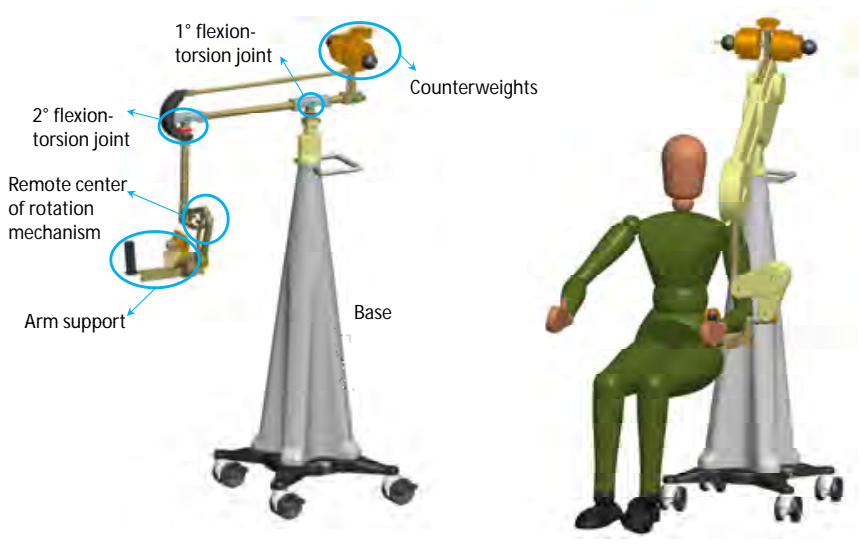

Fig. 8. CAD model of the Trackhold
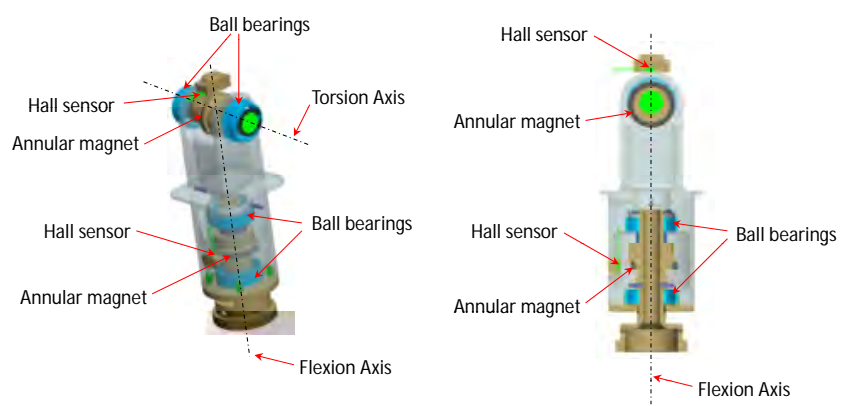

Fig. 9. CAD model of flexion-torsion joint and one of its sections

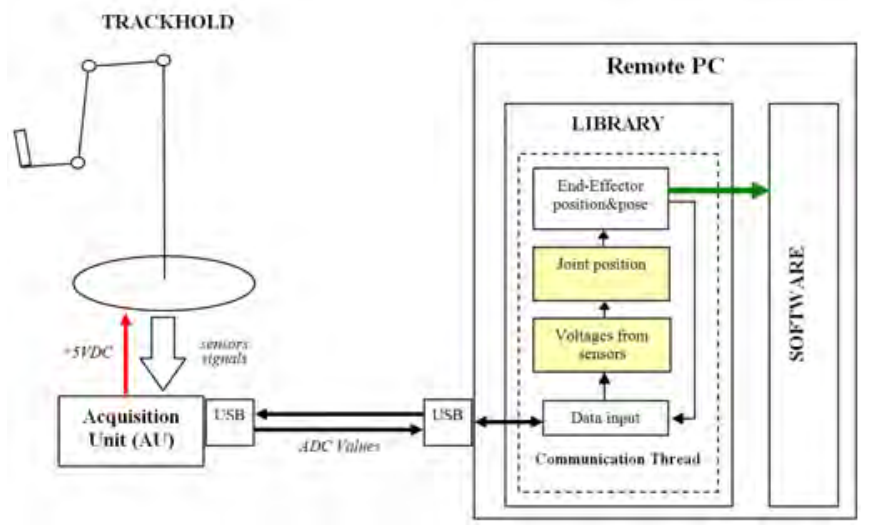

Fig. 10. Architecture of electronics

The structural design of the flexion-torsion joint, basically composed of a metallic frame supporting two pins on ball bearings, is integrated with the design of the sensing architecture. The sensing architecture is based on a recent principle using only one hall-effect sensor and a commercial permanent magnet with simple shape, successfully tested in $[25,26]$. Indeed, for both axes of the joint a NeodymiumIron-Boron permanent magnet is installed in eccentric position with respect to the rotation axis, and a $A 1321$ hall-effect sensor by Allegro, with a typical sensitivity of $5 \mathrm{mV} / \mathrm{G}$ (with $5 \mathrm{~V}$ of power supply), is implemented. The two magnets are annular as to allow electrical cables to pass inside the flexiontorsion joint. The flexion-torsion joint was dimensioned by means of FEM analyses: the final CAD model is shown in Fig. 9.

\subsection{Architecture of the electronics}

The electronics architecture of the Trackhold (Fig. 10) features a remote PC and an Acquisition Unit (AU). The AU is placed inside the base of the Trackhold and receives the signals from the sensorized joints by means of electrical cables passing inside the tubes and the joints of the device. The $\mathrm{AU}$ is composed by:

- Power Unit, providing the voltages required by the position sensors and the components of the AU;

- ADC converter, converting the signals of the sensorized joint from analog to digital;

- Control and Communication Unit, managing the data acquisition and communicating the acquired data to the remote $\mathrm{PC}$ through a USB connection.

The AU (Fig. 11) is powered at $+5 \mathrm{~V}$ via the USB connection. The components of the AU are powered by means of three DC/DC converters model Burr-Brown DCR010505, which tune precisely the output voltage eliminating the typical fluctuations of the USB power. The output voltages are: $+5 \mathrm{~V}$ digital, powering the microcontroller and the digital section of the ADC converters; $\pm 5 \mathrm{~V}$ analog, powering the position sensors and the analog section of the ADC converters. The AU features two analog/digital converter model 


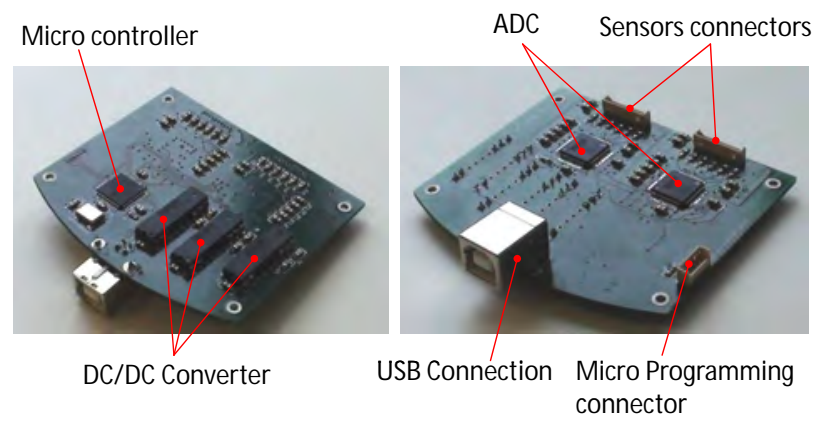

Fig. 11. Acquisition Unit (AU)

Analog Device $A D 7656$, each capable of acquiring 6 input signals. The microcontroller, model PIC18F4455 (by Microchip), controls the acquisition of the signals of the sensors (received via Serial Peripheral Interface SPI), based on the input commands received via USB from the PC. Finally, the acquired signals are sent to the remote PC via USB. Once in the $\mathrm{PC}$, voltage signals are extracted from data input, and the position of the joints is calculated based on voltage signals. The application of the direct kinematics equations allows to obtain the position and orientation of the end effector, which are given to the user interface of the software.

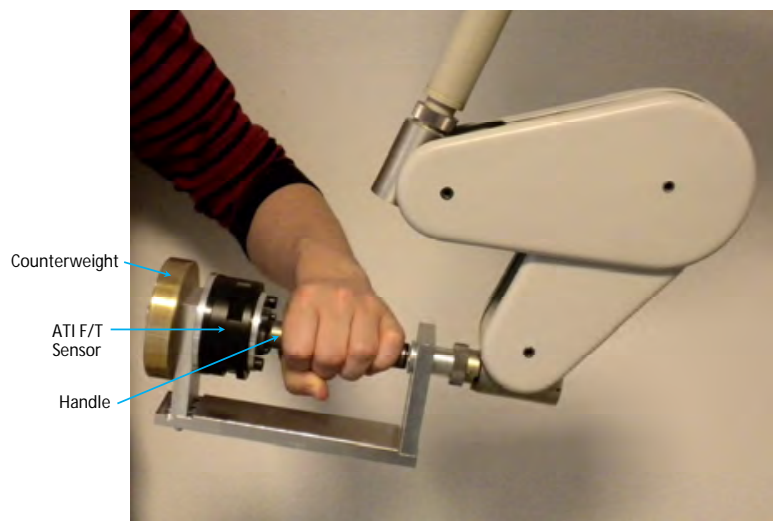

Fig. 12. Detail of the Trackhold with the test end-effector

\section{Experimental dynamic verification}

Once the device was fabricated and assembled, an experimental validation of its dynamic performance was set up. A custom end-effector was designed and fabricated, consisting of a support for housing a handle for the user's hand, a force/torque sensor model ATI $F / T$ Transducer [27] to measure the wrench between user and device, and a suitable counterweight as to maintain the indifferent equilibrium of the Trackhold (Fig. 12). The data acquired from the force/torque sensor (by means of a dedicated acquisition board) were synchronized with the Trackhold joint positions read via USB as described in 4.3. The wrench read by the force/torque sensor was corrected considering the location of the sensor, so to refer the wrench at the level of the CCM point coherently with the theoretical analysis (Section 3).

As a first test of comparison between theoretical and practical results, the protocol used in the theoretical dynamic analysis (Section 3) was repeated, again with a theoretical supporting force of $11.30 \mathrm{~N}$. Figure 13 shows the results of the test for movements on the horizontal plane. Still referring to the $\left\{x_{0}, y_{0}, z_{0}\right\}$ frame (Fig. 3), the experimental support force resulted $11.53 \pm 0.78 \mathrm{~N}$ (axis $z$ ), with disturbances of $1.24 \pm 1.17 \mathrm{~N}$ along axis $x, 1.15 \pm 1.18 \mathrm{~N}$ along axis $y$, and torques below $\approx 0.4 \mathrm{Nm}$ along axis $x$ and below $\approx 0.2 \mathrm{Nm}$ along axes $y$ and $z .^{6}$
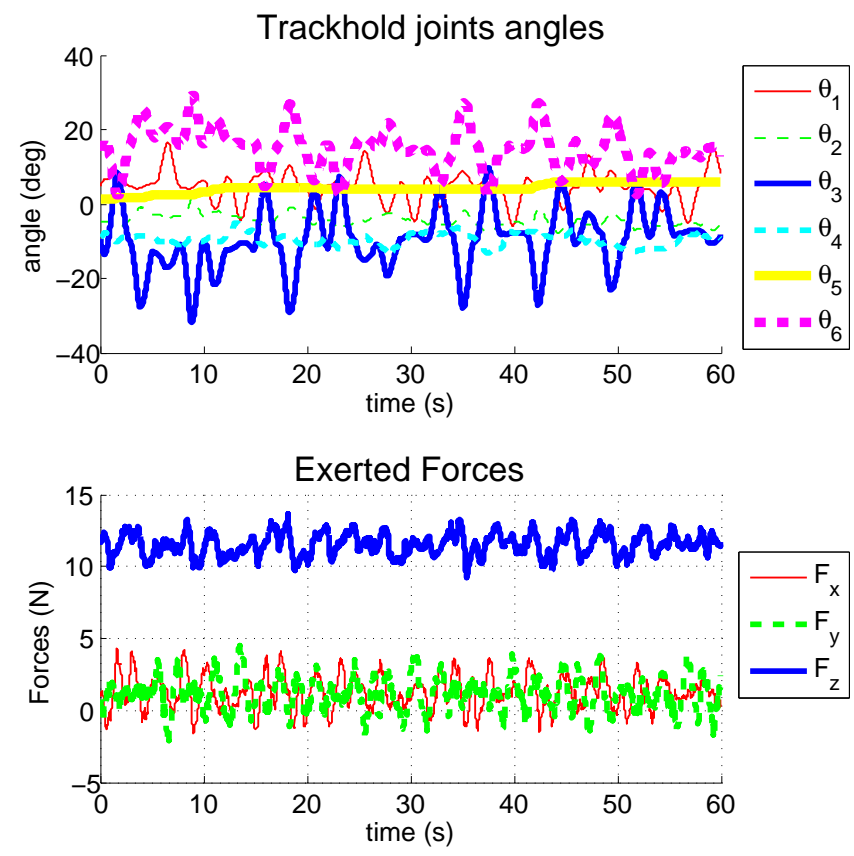

Fig. 13. First experimental test: joint coordinates and measured wrench at the end effector

As a second test, a subject was asked to move the end effector trying to explore the workspace of the device, simulating free 3D reaching, with the same supporting force of the first test $(11.30 \mathrm{~N})$.

Figure 14 shows the results of the test in which several different movements were tried. The experimental support force resulted $11.28 \pm 0.65 \mathrm{~N}$, with disturbance forces below $2 \mathrm{~N}$ and torques below $0.4 \mathrm{Nm}$.

To sum up, the experimental tests confirmed the good dynamic performance of the Trackhold, which provides the desired support force along all the workspace with average errors limited to $\approx 10 \%$ of it. Small discrepancies resulted

\footnotetext{
${ }^{6}$ The disturbance torques can be interpreted as an estimation of how far is the real application point of the force with respect to the theoretical one: approximately, $0.4 \mathrm{Nm}$ (peak value) over $11.5 \mathrm{~N}$ means $3 \mathrm{~cm}$, which is considered negligible with respect to the dimensions of the human arm.
} 

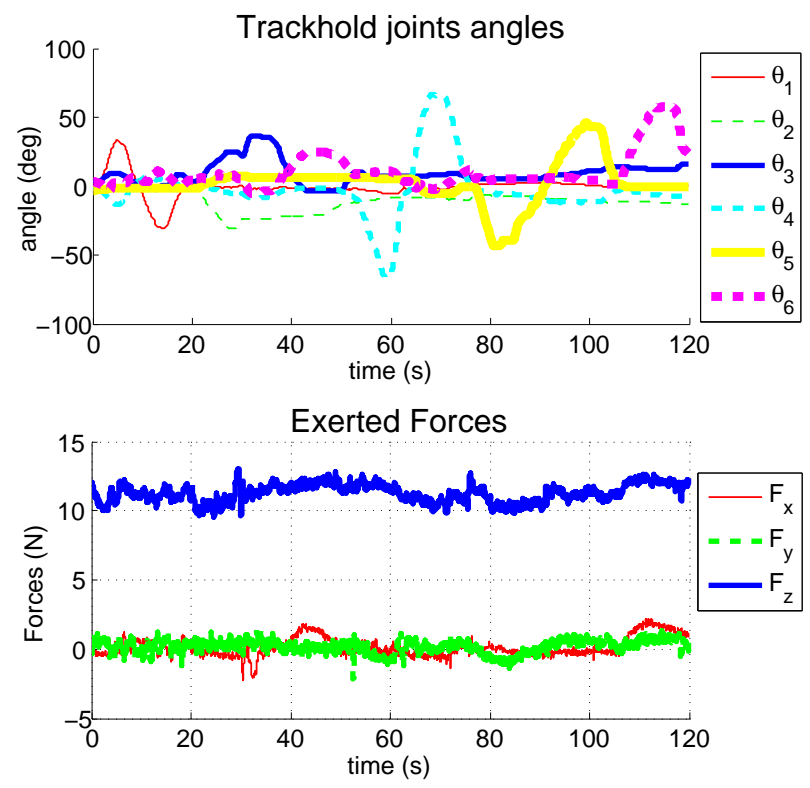

Fig. 14. Second experimental test: joint coordinates and measured wrench at the end effector

between the theoretical dynamic model and the practical behaviour of the device, they might be due to:

- not exact correspondence real device - CAD model;

- not perfect modelling of dynamic effects;

- not perfect mechanical balancing of the device;

- slight orientation error in mounting the force sensor;

- slight errors in the joint angle measurements (affecting the rotation matrix between base frame and end effector, used to convert the sensor measurements in the base frame).

\section{Conclusion}

We proposed a novel passive arm-support device, the Trackhold (Fig. 15). The device was designed with a taskbased approach oriented at supporting users with motor impairments (e.g. after stroke) to practice arm movements without continuous supervision from a rehabilitation therapist. The device supports passively the weight of the human arm independently of the configuration, exploiting a pantograph mechanism and a proper tunable counterweight. The joint positions are sensorized as to track position and orientation of the user's arm, making the device suitable also for rehabilitation exercises where the user interacts with virtual environments. The design of the device was carried out taking into account the requirements of high transparency, high resolution, high refresh rate, scalable workspace, and low cost. $^{7}$

The theoretical kinematic and dynamic analyses showed that the Trackhold has a good performance even in dynamic conditions. Then, the device has been fabricated and the im-

\footnotetext{
${ }^{7}$ The cost of the device is expected to be around 20000 Euro.
}

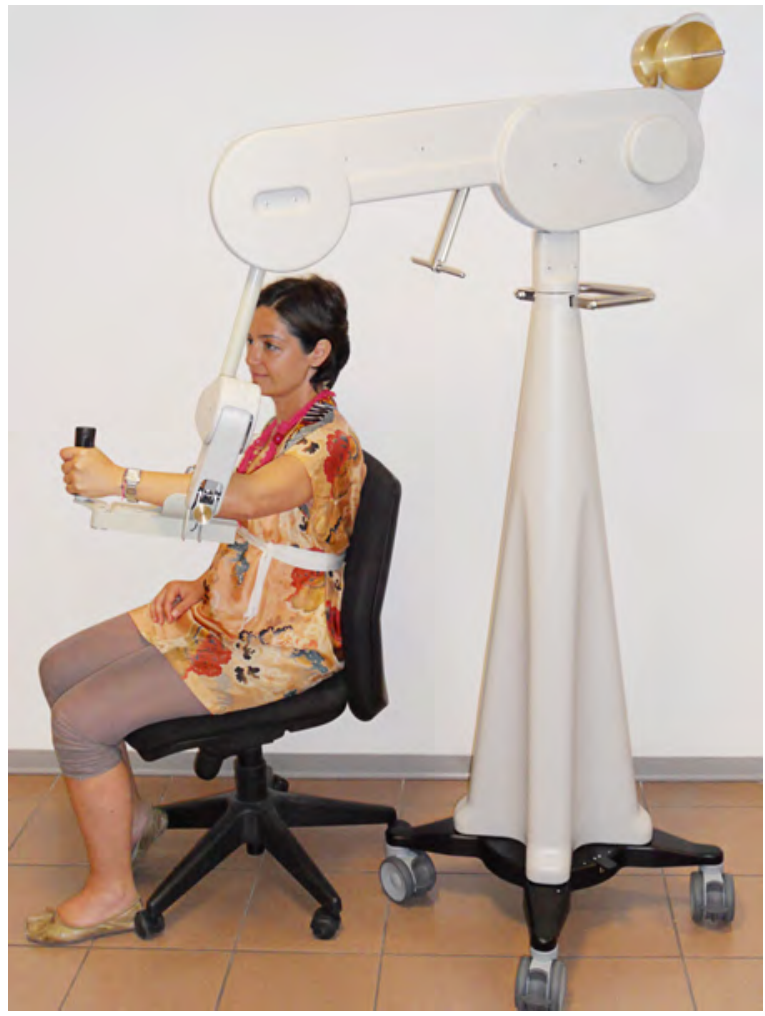

Fig. 15. A user wearing the Trackhold

plemented design solutions worked correctly, guaranteeing the desired support force throughout all the workspace with less than $\approx 10 \%$ average error in dynamic conditions. Future investigations will be dedicated to tests of the device with motor impaired people needing rehabilitation, i.e. the subjects for which the use of the device is intended. Lastly, the Trackhold is currently being engineered for commercial purposes.

\section{References}

[1] Reinkensmeyer, D. J., and Housman, S. J., 2007. "'” if i can't do it once, why do it a hundred times?": Connecting volition to movement success in a virtual environment motivates people to exercise the arm after stroke". In Virtual Rehabilitation, 2007, IEEE, pp. 44-48.

[2] Kwakkel, G., van Peppen, R., Wagenaar, R. C., Dauphinee, S. W., Richards, C., Ashburn, A., Miller, K., Lincoln, N., Partridge, C., Wellwood, I., et al., 2004. "Effects of augmented exercise therapy time after stroke a meta-analysis". Stroke, 35(11), pp. 2529-2539.

[3] Byl, N. N., Pitsch, E. A., and Abrams, G. M., 2008. "Functional outcomes can vary by dose: learning-based sensorimotor training for patients stable poststroke". Neurorehabilitation and neural repair, 22(5), pp. 494504.

[4] Prange, G. B., Jannink, M. J., Groothuis-Oudshoorn, C. G., Hermens, H. J., IJzerman, M. J., et al., 2006. "Systematic review of the effect of robot-aided therapy on recovery of the hemiparetic arm after stroke". Jour- 
nal of rehabilitation research and development, 43(2), p. 171.

[5] Lum, P. S., Taub, E., Schwandt, D., Postman, M., Hardin, P., and Uswatte, G., 2004. "Automated constraint-induced therapy extension (autocite) for movement deficits after stroke". Journal of rehabilitation research and development, 41(3), pp. 249-258.

[6] Sanchez, R. J., Liu, J., Rao, S., Shah, P., Smith, R., Rahman, T., Cramer, S. C., Bobrow, J. E., and Reinkensmeyer, D. J., 2006. "Automating arm movement training following severe stroke: functional exercises with quantitative feedback in a gravity-reduced environment". Neural Systems and Rehabilitation Engineering, IEEE Transactions on, 14(3), pp. 378-389.

[7] Stienen, A. H., Hekman, E. E., van der Kooij, H., Ellis, M. D., and Dewald, J. P., 2009. "Aspects of weight-support mechanisms in rehabilitation robotics". In World Congress on Medical Physics and Biomedical Engineering, September 7-12, 2009, Munich, Germany, Springer, pp. 392-394.

[8] Garrec, P., Friconneau, J., Measson, Y., and Perrot, Y., 2008. "Able, an innovative transparent exoskeleton for the upper-limb". In Intelligent Robots and Systems, 2008. IROS 2008. IEEE/RSJ International Conference on, IEEE, pp. 1483-1488.

[9] Frisoli, A., Borelli, L., Montagner, A., Marcheschi, S., Procopio, C., Salsedo, F., Bergamasco, M., Carboncini, M., Tolaini, M., and Rossi, B., 2007. "Arm rehabilitation with a robotic exoskeleleton in virtual reality". In Rehabilitation Robotics, 2007. ICORR 2007. IEEE 10th International Conference on, IEEE, pp. 631-642.

[10] Gijbels, D., Lamers, I., Kerkhofs, L., Alders, G., Knippenberg, E., Feys, P., et al., 2011. "The armeo spring as training tool to improve upper limb functionality in multiple sclerosis: a pilot study". Journal of neuroengineering and rehabilitation, 8(5), p. 5.

[11] Herder, J., 2005. "Development of a statically balanced arm support: Armon". In Rehabilitation Robotics, 2005. ICORR 2005. 9th International Conference on, IEEE, pp. 281-286.

[12] Prange, G., Jannink, M., Stienen, A., Van der Kooij, H., Ijzerman, M., and Hermens, H., 2009. "Influence of gravity compensation on muscle activation patterns during different temporal phases of arm movements of stroke patients". Neurorehabilitation and neural repair.

[13] Kloosterman, M., Snoek, G. J., Kouwenhoven, M., Nene, A. V., and Jannink, M., 2010. "Influence of gravity compensation on kinematics and muscle activation patterns during reach and retrieval in subjects with cervical spinal cord injury: an explorative study". J Rehabil Res Dev, 47(7), pp. 617-28.

[14] Ellis, M., Sukal, T., DeMott, T., and Dewald, J., 2007. "Act 3d exercise targets gravity-induced discoordination and improves reaching work area in individuals with stroke". In Rehabilitation Robotics, 2007. ICORR 2007. IEEE 10th International Conference on, IEEE, pp. 890-895.

[15] Kisner, C., and Colby, L. A., 2012. Therapeutic exer- cise: foundations and techniques. FA Davis.

[16] Stienen, A. H., Hekman, E. E., Prange, G. B., Jannink, M. J., van der Helm, F. C., and van der Kooij, H., 2009. "Freebal: design of a dedicated weight-support system for upper-extremity rehabilitation". Journal of Medical Devices, 3(4), p. 041009.

[17] Lenzo, B., Frisoli, A., Salsedo, F., and Bergamasco, M., 2014. "New gravity balancing technique and hybrid actuation for spatial serial manipulators". In Advances in Robot Kinematics. Springer, pp. 419-427.

[18] Sciavicco, L., and Villani, L., 2009. Robotics: modelling, planning and control. Springer.

[19] Clauser, C. E., McConville, J. T., and Young, J. W., 1969. Weight, volume, and center of mass of segments of the human body. Tech. rep., DTIC Document.

[20] Hartenberg, R., and Denavit, J. "1964, kinematic synthesis of linkages, mcgraw-hill, new york".

[21] Klopčar, N., and Lenarčič, J., 2005. "Kinematic model for determination of human arm reachable workspace". Meccanica, 40(2), pp. 203-219.

[22] Xu, P., Jingjun, Y., and Guanghua, B. S. Z., 2007. "Enumeration and type synthesis of one-dof remotecenter-of-motion mechanisms". In The 12th world congress in mechanism an machine science.

[23] Fontana, M., Fabio, S., Marcheschi, S., and Bergamasco, M., 2013. "Haptic hand exoskeleton for precision grasp simulation". Journal of Mechanisms and Robotics, 5(4), p. 041014.

[24] NaturalPoint OptiTrack, Accessed September 4, 2014. http://www.naturalpoint.com/optitrack/.

[25] Tarri, F., Fontana, M., Salsedo, F., Marcheschi, S., and Bergamasco, M., 2009. "Modular weight-balanced mechanical tracker for portable haptics". In Robotics and Automation, 2009. ICRA'09. IEEE International Conference on, IEEE, pp. 1473-1478.

[26] Fontana, M., Salsedo, F., and Bergamasco, M., 2013. "Novel magnetic sensing approach with improved linearity". Sensors, 13(6), pp. 7618-7632.

[27] ATI Force/Torque sensors, Accessed September 4, 2014. http://www.ati-ia.com/products/ft/sensors.aspx.

[28] Bergamasco, M., Fontana, M., and Salsedo, F., 2012. Device to relieve the articular efforts resulting from the weight of a human limb, May 31. WO Patent App. PCT/IB2011/053,986.

\section{Appendix A: Detailed dynamic model of the Trackhold}

The dynamics of the device is described by Eq. 9. The matrices $\mathbf{B}$ and $\mathbf{C}$ and the vector $\mathbf{G}$ were computed according to [18], referring again to the frame $\left\{x_{0}, y_{0}, z_{0}\right\}$ :

$$
\begin{aligned}
\mathbf{B}(\mathbf{q}) & =\sum_{i=1}^{n}\left(m_{i} \mathbf{J}_{P_{i}}(\mathbf{q})^{T} \mathbf{J}_{P_{i}}(\mathbf{q})+\right. \\
& \left.\left.+\mathbf{J}_{O_{i}}(\mathbf{q})^{T} \mathbf{R}_{l_{i}}^{0}(\mathbf{q}) \mathbf{J}_{G_{i}}(\mathbf{q}) \mathbf{R}_{l_{i}}^{0}(\mathbf{q})^{T}\right] \mathbf{J}_{O_{i}}(\mathbf{q})\right)
\end{aligned}
$$




$$
\begin{gathered}
c_{i j}=\sum_{i=1}^{n} \Gamma_{j k}^{i} \dot{q}_{k} \\
\mathbf{G}(\mathbf{q})=-\mathbf{g}^{T} \sum_{i=1}^{n} m_{i} \mathbf{p}_{G_{i}}(\mathbf{q})
\end{gathered}
$$

being:

- $n$ the number of links;

- $\Gamma_{j k}^{i}=\frac{1}{2}\left(\frac{\partial b_{i j}}{\partial q_{k}}+\frac{\partial b_{i k}}{\partial q_{j}}-\frac{\partial b_{j k}}{\partial q_{i}}\right)$ the Christoffel symbols of the first type;

- $b_{i j}$ and $c_{i j}$ the generic terms of $\mathbf{B}(\mathbf{q})$ and $\mathbf{C}(\mathbf{q})$, respectively;

- $m_{i}$ the mass of link $i$

- $\mathbf{J}_{G_{i}}$ the inertia matrix of link $i$, computed with respect to a frame located in the center of mass of link $i$ and having axes oriented as the frame $\left\{x_{i}, y_{i}, z_{i}\right\}$ (Fig. 3);

- $\mathbf{p}_{G_{i}}$ the position of the center of mass of link $i$ with respect to the frame $\left\{x_{0}, y_{0}, z_{0}\right\}$

- g the gravity acceleration vector with respect to the frame $\left\{x_{0}, y_{0}, z_{0}\right\}$;

- $\mathbf{R}_{l_{i}}^{0}$ the rotation matrix from the frame $\left\{x_{0}, y_{0}, z_{0}\right\}$ to the frame $\left\{x_{i}, y_{i}, z_{i}\right\}$;

- $\mathbf{J}_{P_{i}}$ the positional Jacobian related to the center of gravity of link $i$

- $\mathbf{J}_{O_{i}}$ the orientational Jacobian related to the center of gravity of link $i$.

The inertia properties were computed by means of a CAD model of the Trackhold, in which all the links were modelled as hollow tubes made of aluminum. The inertia properties of the pantograph were also considered by means of proper $\mathbf{J}_{P_{i}}$ and $\mathbf{J}_{O_{i}}$ terms, considering the dependence of the pantograph joints coordinates with respect to the six lagrangian coordinates. 